Article

\title{
Negative Thermal Expansivity of Ice: Comparison of the Monatomic mW Model with the All-Atom TIP4P/2005 Water Model
}

\author{
Muhammad Mahfuzh Huda ${ }^{1} \mathbb{D}$, Takuma Yagasaki ${ }^{2}\left(\mathbb{D}\right.$, Masakazu Matsumoto $^{2} \mathbb{D}$ \\ and Hideki Tanaka ${ }^{2, *(\mathbb{D})}$ \\ 1 Graduate School of Natural Science and Technology, Okayama University, Tsushima-naka, \\ Okayama 700-8530, Japan; hudamahfuzh@gmail.com \\ 2 Research Institute for Interdisciplinary Science, Okayama University, Tsushima-naka, \\ Okayama 700-8530, Japan; t.yagasaki@gmail.com (T.Y.); vitroid@gmail.com (M.M.) \\ * Correspondence: htanakaa@okayama-u.ac.jp; Tel.: +81-86-251-7769
}

Received: 28 March 2019; Accepted: 12 May 2019; Published: 14 May 2019

check for updates

\begin{abstract}
We calculate the thermal expansivity of ice I for the monatomic mW model using the quasi-harmonic approximation. It is found that the original $\mathrm{mW}$ model is unable to reproduce the negative thermal expansivity experimentally observed at low temperatures. A simple prescription is proposed to recover the negative thermal expansion by re-adjusting the so-called tetrahedrality parameter, $\lambda$. We investigate the relation between the $\lambda$ value and the Grüneisen parameter to explain the origin of negative thermal expansion in the $\mathrm{mW}$ model and compare it with an all-atom water model that allows the examination of the effect of the rotational motions on the volume of ice.
\end{abstract}

Keywords: Negative thermal expansivity; mW model; Grüneisen parameter

\section{Introduction}

Liquid water freezes into ice I under ambient pressure. One of interesting characteristics of ice I is its negative thermal expansivity at low temperatures [1-5]. This was also recovered by theoretical study [6]. The thermal expansivity of a crystal can be related to the so-called Grüneisen parameter. For a mode with frequency $v$, this parameter is defined as $\gamma=-(\mathrm{d} \ln v / \mathrm{d} \ln V)$ [7]. The quantity is positive for stretching modes. This is evident from the sharp increase in the potential energy with a decrease in the bond length caused by the Pauli exclusion principle. By contrast, the Grüneisen parameter can be negative for bending modes. The negative thermal expansivity is realized when the Grüneisen parameter of the bending modes is negative and the positive contribution from the stretching modes is small enough.

The Gibbs energy calculation with the quasi-harmonic approximation is a useful computational approach to examine the thermal properties of crystals [8]. This method allows for the calculation of the temperature dependence of the equilibrium volume of a crystal, from which one can obtain the thermal expansivity. It also enables one to calculate the components of the thermal expansivity, that is, the heat capacity and the Grüneisen parameters of individual intermolecular vibrational modes. The quality of the results depends strongly on the employed force field. Excellent results can be obtained from ab initio methods considering a high level electron correlation with a large basis set, but such calculations are quite time-consuming and may therefore not be suitable for the treatment of ice I, for which the system size and the number of examined configurations should be large enough to take the hydrogen-disorder into account [9]. In addition, properties of molecular solids, including ice, are sensitive to van der Waals interactions while empirical models to correct the van der Waals interactions for density functional theory (DFT) methods are not specialized for 
ice [10]. At this point, classical force fields modelled to reproduce properties of ice I work better than $a b$ initio force fields, except for the cleavage of chemical bonds. Of course, the ab initio calculation and the path integral method are useful for examining the isotope effects and for taking into account the anharmonic contributions [11-13]. Some discussions on the most effective method among these types of calculations has been conducted [14].

Here, we take advantage of the excellent reproducibility of classical force fields parameterized for liquid water and ice polymorphs. In passing, this kind of approach costs rather little, so that we can handle not only a larger number of molecules (say 1000) in the simulation cell but also many hydrogen-disordered structures, such as 100 different structures. The TIP4P/2005 model is one such classical force field [15]. A water molecule consists of one oxygen atom without charge, two positively charged hydrogen atoms, and one negatively charged site without mass. The oxygen atom interacts with oxygen atoms of other molecules via the Lennard-Jones (LJ) potential. This model reproduces the phase diagram of ice for a wide range of pressure except for the very high pressure region [16-19]. TIP4P/2005 is a re-parameterized TIP4P model [20]. It has been demonstrated that the thermal expansivity of ice I is negative at temperatures that are under $60 \mathrm{~K}$ when the original TIP4P model is employed [6]. A similar method was applied to ice II and III [21].

The monatomic water $(\mathrm{mW})$ model is a different type of empirical model for water [22]. This is a single site water model, and the intermolecular interactions are described by the sum of the short-ranged pair and three-body interactions. The computational cost of the $\mathrm{mW}$ model is quite low because the number of interaction sites is reduced and because the long-range interactions are omitted. Nevertheless, this model reproduces various properties of ice I well, such as the melting point. It has been used frequently in molecular dynamics (MD) studies of nucleation and growth of not only ice I but also clathrate hydrates $[23,24]$. The $\mathrm{mW}$ model was developed on the basis of the Stillinger-Weber silicon model [25]. Although crystalline Si exhibits negative thermal expansivity, the Stillinger-Weber Si potential does not reproduce this anomaly [26].

The negative thermal expansivity of ice Ih has recently been measured in $a$ and $c$ lattice directions. Both measurements show an anisotropy in the thermal expansion between the $a$-axis and $c$-axis [3-5]. The temperature of the minimum thermal expansivity in those experiments shifts toward a low temperature, around $30 \mathrm{~K}$. The negative thermal expansivity is observed for some crystals with open network structures, such as silicon, quartz, and silica zeolites. This fact suggests that the preference of a molecule for a regular tetrahedral arrangement, which is the origin of the low density of ice, is an important factor for the negative thermal expansivity [27]. In the $\mathrm{mW}$ model, the preference is implemented by a parameter in the three body interaction term. In this study, we calculate the thermal expansivity of ice I using the quasi-harmonic approximation and examine the effect of this tetrahedrality parameter on the thermal expansivity. We also calculate the thermal expansivity for the TIP4P/2005 model. The vibrational modes can be classified into the translation-dominant and rotation-dominant modes because it is an all-atom model. We demonstrate that the coupling between the two types of modes plays a vital role for the negative thermal expansivity of ice.

Our aim in this work is to explore how the $\mathrm{mW}$ model works in low temperatures by examining the thermal expansivity of ice. The original potential for the Si atom has been made, and the negative thermal expansion is recovered for amorphous Si only [28]. Therefore, it is of interest to examine if the $\mathrm{mW}$ model can reproduce the negative thermal expansivity and how to revise it to lead to its better reproducibility.

\section{Method}

\subsection{Force Field Models and Structure of Ice}

The potential energy of the $\mathrm{mW}$ model is the sum of the pairwise and three-body interactions [22]:

$$
E=\varepsilon \sum_{\mathrm{i}} \sum_{\mathrm{j}>\mathrm{i}} \phi_{2}\left(r_{i j}\right)+\sum_{\mathrm{i}} \sum_{\mathrm{j} \neq \mathrm{i}} \sum_{\mathrm{k}>\mathrm{j}} \phi_{3}\left(r_{i j}, r_{i k}, \theta_{i j k}\right),
$$


with

$$
\phi_{2}(r)=A\left[B\left(\frac{\sigma}{r}\right)^{4}-1\right] \exp \left(\frac{\sigma}{r-a \sigma}\right)
$$

and

$$
\phi_{3}(r, s, \theta)=\lambda\left[\cos \theta-\cos \theta_{0}\right]^{2} \exp \left(\frac{\gamma \sigma}{r-a \sigma}\right) \exp \left(\frac{\gamma \sigma}{s-a \sigma}\right) .
$$

The parameters are given in Table 1. The interaction is short-ranged: a molecule interacts with a different molecule only when the distance between them is shorter than $a \sigma=0.43065 \mathrm{~nm}$. The three-body term is zero when the O-O-O angle is the tetrahedral angle of $\theta_{0}=109.47^{\circ}$, and otherwise it is positive. Therefore, the preference for tetrahedral arrangements increases with the increase of the coefficient of the three body term, $\lambda$. In the $\mathrm{mW}$ model, this tetrahedrality parameter is set to 23.15 to reproduce various properties of ice I and liquid water [22,24,29].

Table 1. Parameters of the mW model [22].

\begin{tabular}{cc}
\hline$A$ & 7.049556277 \\
\hline$B$ & 0.6022245584 \\
\hline$\gamma$ & 1.2 \\
\hline$a$ & 1.8 \\
\hline$\lambda$ & 23.15 \\
\hline$\theta_{0}$ & $109.47^{\circ}$ \\
\hline$\sigma$ & $0.23925 \mathrm{~nm}$ \\
\hline$\varepsilon$ & $25.895 \mathrm{~kJ} \mathrm{~mol}^{-1}$ \\
\hline
\end{tabular}

The TIP4P/2005 model is an all-atom rigid model for water [15]. The intermolecular interactions are described by pairwise additive Coulomb and LJ interactions. A negatively charged site without mass, $\mathrm{M}$, is located on the bisector of the $\mathrm{H}-\mathrm{O}-\mathrm{H}$ angle with an O-M distance of $0.01546 \mathrm{~nm}$. The O-H distance and the $\mathrm{H}-\mathrm{O}-\mathrm{H}$ angle are $0.09572 \mathrm{~nm}$ and $104.52^{\circ}$, respectively. The partial charge on each hydrogen atom is $0.5564 e$. The LJ parameters of the oxygen atom are $\sigma_{O}=0.31589 \mathrm{~nm}$ and $\varepsilon_{O}=0.774912 \mathrm{~kJ} \mathrm{~mol}^{-1}$.

The structure of ice in this study is ice Ic, consisting of 1000 water molecules. A hundred hydrogen-disordered ice structures without net polarization are generated for the TIP4P/2005 model by the GenIce tool [30]. For ices with the $\mathrm{mW}$ model, oxygen atoms are simply placed on the corresponding lattice sites. The calculations are performed for numerous densities. The shape of the cell is kept cubic throughout the expansion or compression. Periodic boundary conditions are applied to ensure that the molecules are in the bulk phase without any surface effect.

\subsection{Quasi-Harmonic Approximation}

The Helmholtz energy of ice can be expressed as:

$$
A(T, V)=U_{q}(V)+F(T, V)-T S_{c},
$$

where $U_{q}(V)$ is the potential energy at $0 \mathrm{~K}, F(T, V)$ is the vibrational free energy, and $S_{c}$ is the configurational entropy. The vibrational free energy consists of the harmonic and anharmonic contributions. The anharmonic part can be ignored at low temperatures at which the potential energy surface is regarded to be parabolic in any direction. The harmonic vibrational free energy is given by:

$$
F_{\mathrm{h}}(T, V)=k_{\mathrm{B}} T \sum_{i} \ln \left[2 \sinh \left(\frac{h v_{i}}{2 k_{\mathrm{B}} T}\right)\right]
$$


where $h$ is the Planck constant, $k_{\mathrm{B}}$ is the Boltzmann constant, and $v_{i}$ is the frequency of the $i$-th normal mode. The steepest descent method is employed to obtain the crystalline configuration at $T=0 \mathrm{~K}$ and its potential energy, $U_{q}(V)$. Then, we calculate the mass-weighted Hessian matrix and diagonalize it to obtain the normal mode frequencies. The equilibrium volume, $\langle V\rangle$, at a given pressure, $p$, and at a temperature, $T$, is the volume that minimizes the following thermodynamic potential:

$$
\Psi(V ; T, p)=A(T, V)+p V .
$$

The pressure is set to $0.1 \mathrm{MPa}$ in all of the calculations. The Gibbs free energy is given by:

$$
G(T, p)=\Psi(\langle V\rangle ; T, p) .
$$

\section{Results}

As will be shown below, the thermal expansivity is connected with the frequencies of the intermolecular vibrations via the Grüneisen parameter and the heat capacity. Therefore, some vibrational modes play a significant role in the appearance of its anomaly under cryogenic conditions. The distribution, known as the density of states (DOS), reveals the vibrational characteristics of ice. The calculated DOS resembles that of neutron scattering experiments, as shown in Figure $1[9,31]$. The DOS of the TIP4P/2005 model has two peaks. The modes in the lower frequency region of up to $400 \mathrm{~cm}^{-1}$ originate mostly from the translational motions, while the modes between 500 and $1000 \mathrm{~cm}^{-1}$ are associated mostly with the rotational (librational) motions. The frequency range of $\mathrm{mW}$ ice is limited to $250 \mathrm{~cm}^{-1}$ because all of the modes arise from the translational motions. The calculated DOS for TIP4P/2005 ice reproduces the overall feature of the experimental DOS. There are two distinct peaks in the low frequency region in both experimental and TIP4P/2005 ice. The DOSs in the rotation-dominant region agree roughly with each other. The $\mathrm{mW}$ model also has two distinct peaks in the low frequency region. The peak position of the higher one is fairly different from either of the other two. However, the position of the lower peak agrees with that of the TIP4P/2005 ice model and of the experimental one. Since the modes in this low frequency region, which corresponds to the O-O-O bending, play a central role in giving rise to a negative thermal expansivity [6], the $\mathrm{mW}$ model or its variants could reproduce the negative thermal expansivity.

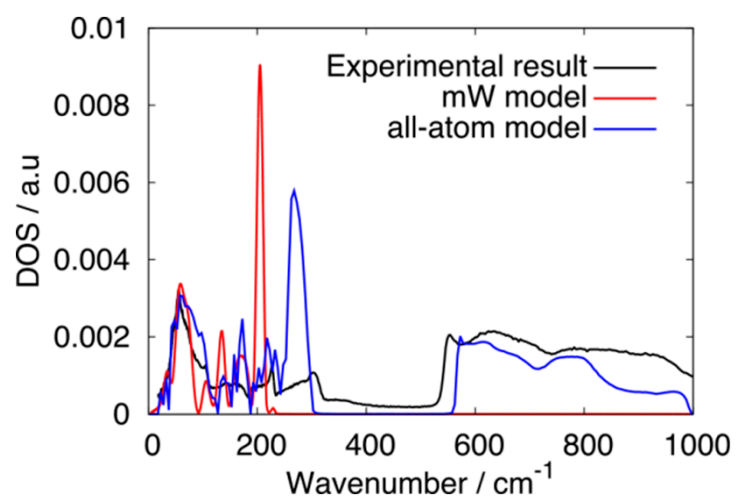

Figure 1. Densities of state of ice Ic calculated with the $\mathrm{mW}$ model $(\lambda=23.15)$ and that of the all-atom model. The experimental neutron scattering data for ice Ih is also shown [31].

Although the $\mathrm{mW}$ model was developed for ice I, we attempt to use this model for other ice phases. The thermodynamic potential, $A+p V$, is plotted against the molar volume for several ice phases in Figure 2. The equilibrium molar volume, $\langle V\rangle$, is the volume at which the thermodynamic potential is minimized. The equilibrium molar volume is reasonably obtained for ice Ic, Ih, II, and III. The curves of ices II and III reside totally inside the parabola of ice I, meaning that they cannot be the most stable phase at atmospheric pressure. When we elevate the pressure to $300 \mathrm{MPa}$, ice II and III can 
be stable relative to ice Ih with the $\mathrm{mW}$ model. We fail to obtain a smooth curve of the thermodynamic potential for ice VI. Probably, the deviation from the perfect tetrahedral coordination is too large to describe this ice structure through the $\mathrm{mW}$ model. The octahedral coordination of ice VII is also out of the applicable range of the force field model. The octahedral coordination does not mean that there are eight strong bonds for a molecule. It is impossible to distinguish four hydrogen bonding molecules out of the eight neighbors in the $\mathrm{mW}$ model because of the absence of the hydrogen atoms. Another limitation is that the $\mathrm{mW}$ model cannot describe the difference between hydrogen-disordered ice and its hydrogen-ordered counterpart. Thus, there is no way to distinguish, for example, ice Ih from ice XI.

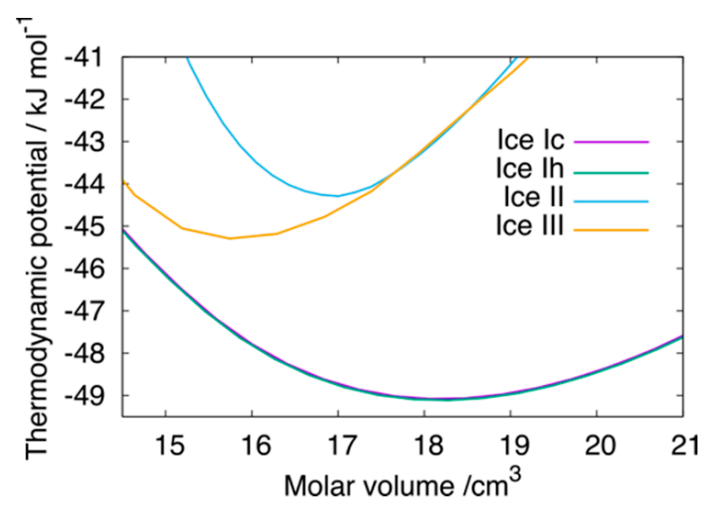

Figure 2. Thermodynamics potential, $A+p V$, for several ice structures at $T=20 \mathrm{~K}$, obtained with the $\mathrm{mW}$ model. The curve for ice Ic overlaps completely with that of ice Ih.

The thermodynamic potential of Ic is the same as that of Ih for the mW model in the framework of the harmonic approximation, although Ih is slightly more stable than Ic experimentally. Because the interaction range is limited to the nearest four neighbors and they form an ideal tetrahedral arrangement both in ice Ih and Ic, no difference between them is expected for $U_{q}$ at a fixed density. No practical difference in the vibrational free energy is found. The free energy from the anharmonic vibrations may give rise to the difference at high temperatures. Hereafter, we will not concern ourselves with the stability difference and will examine only ice Ic.

The equilibrium molar volume for ice Ic is plotted in Figure 3 as a function of temperature. It is evident that the thermal expansivity at low temperatures cannot be reproduced using the original $\mathrm{mW}$ model $(\lambda=23.15)$. In addition, the molar volume of $18.19 \mathrm{~cm}^{3} \mathrm{~mol}^{-1}$ is much smaller than the experimental value [3]. Figure 3 shows the volumes calculated with different $\lambda$ values. We find that the negative thermal expansivity is successfully reproduced when the $\lambda$ value is small enough, though only qualitatively.

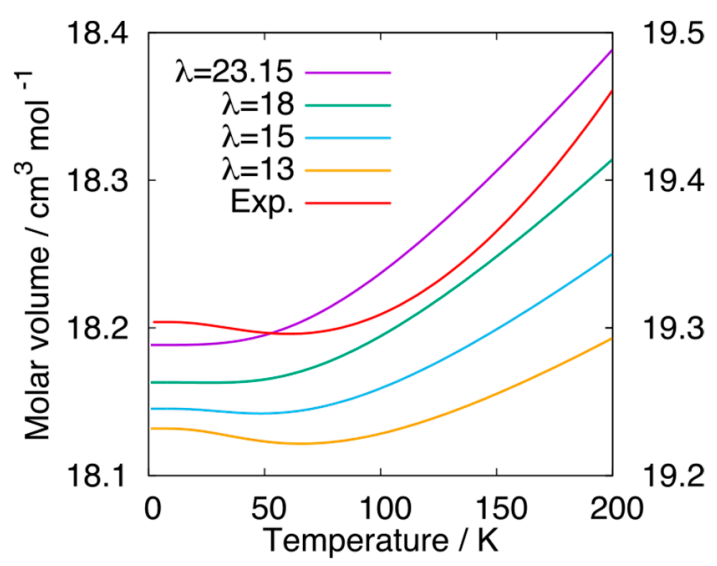

Figure 3. Molar volume of ice Ic as a function of temperature for the $\mathrm{mW}$ model, calculated with several $\lambda$ values. The molar volume of ice Ih from the experimental data is also shown (red, right axis) [3]. 
The equilibrium volume depends substantially on the tetrahedrality parameter, $\lambda$. A smaller $\lambda$ makes the hydrogen bond network more flexible and leads to a more compact packing, while the tetrahedral coordination remains. As shown in Figure 4, the peaks in the DOS shift to a lower frequency as a result of the increase in flexibility due to the decrease in $\lambda$ from 23.15 to 13 . Figure 5 plots the thermal expansivity against the temperature. The critical $\lambda$ value to recover the negative thermal expansivity lies around 18.

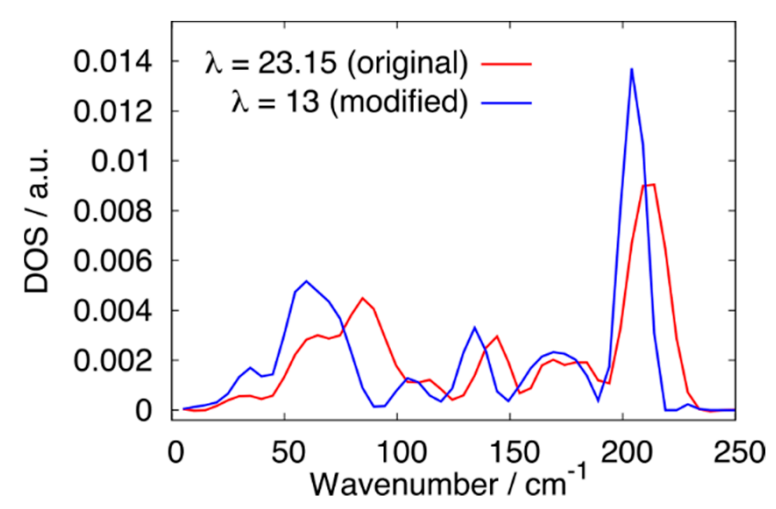

Figure 4. DOSs for ice Ic of the $\mathrm{mW}$ model with the original and modified $\lambda$ values.

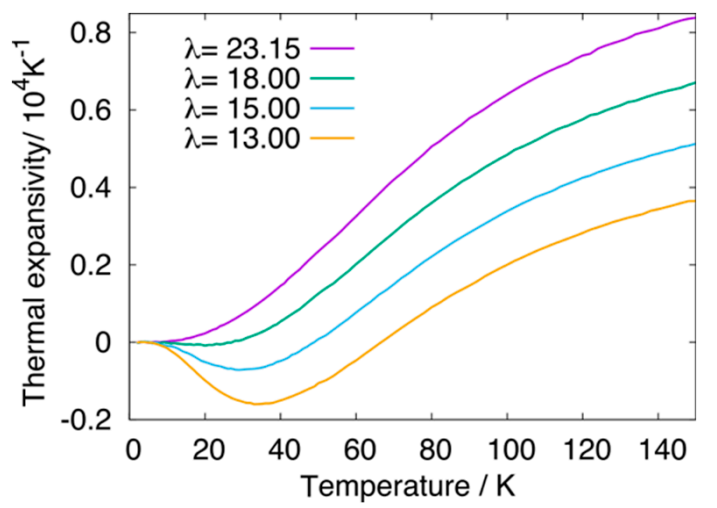

Figure 5. Thermal expansivities of ice Ic calculated using the $\mathrm{mW}$ model with $\lambda=23.15$ (purple line), 18.00 (green line), 15.00 (cyan line), and 13.00 (yellow line).

\section{Discussion}

We calculate the temperature dependence of the molar volume for both the TIP4P/2005 model and the $\mathrm{mW}$ model. The purple curve in Figure 6 is the molar volume of ice Ic plotted against the temperature calculated with the all-atom TIP4P/2005 model. The value of $20.05 \mathrm{~cm}^{3} \mathrm{~mol}^{-1}$ is close to the experimental value for ice $\mathrm{Ih}, 19.3 \mathrm{~cm}^{3} \mathrm{~mol}^{-1}$ [3]. Figure 7 shows the thermal expansivity. The thermal expansivity of the TIP4P/2005 model is negative below $60 \mathrm{~K}$, which is in agreement with the experimental result. We examine the effect of the coupling between the rotational and translational modes on the volume and its temperature dependence. The Hessian matrix, K, can be expressed as:

$$
\mathbf{K}=\left(\begin{array}{ll}
\mathbf{K}_{t t} & \mathbf{K}_{t r} \\
\mathbf{K}_{r t} & \mathbf{K}_{r r}
\end{array}\right)
$$

where $t$ and $r$ denote the translational and rotational components, respectively. We obtain a matrix $\mathbf{K}_{b}$ by removing the rotational-translational coupling elements, $\mathbf{K}_{t r}$ and $\mathbf{K}_{r t}$.

$$
\mathbf{K}_{b}=\left(\begin{array}{cc}
\mathbf{K}_{t t} & 0 \\
0 & \mathbf{K}_{r r}
\end{array}\right)
$$


The thermodynamic properties without the rotational-translational coupling are calculated from the mode frequencies obtained by the diagonalization of the matrix $\mathbf{K}_{b}$. Figure 6 shows that the volume of ice only slightly increases due to the removal of the coupling. However, the negative thermal expansivity disappears completely, as shown in Figure 7. It is also possible to eliminate the contributions from the rotational modes, as well as those from the rotational-translational coupling, by removing $\mathbf{K}_{r r}$ in Equation (9). The volume calculated only from the translational motion is $19.383 \mathrm{~cm}^{3} \mathrm{~mol}^{-1}$, much smaller than the original value of the TIP4P/2005 model, $20.05 \mathrm{~cm}^{3} \mathrm{~mol}^{-1}$. Notwithstanding this, both the mW model and the TIP4P family reproduce the volume of liquid water fairly well [16], although there is a large discrepancy in the volume of ice I. The underestimation of the volume for the $\mathrm{mW}$ model may originate from the absence of the rotational modes. The shift to a higher frequency side upon compression in the TIP4P/2005 model is observed for the modes associated with the rotational motions, which is not shown but is a normal behavior against pressurization. This causes a decrease in the volume.

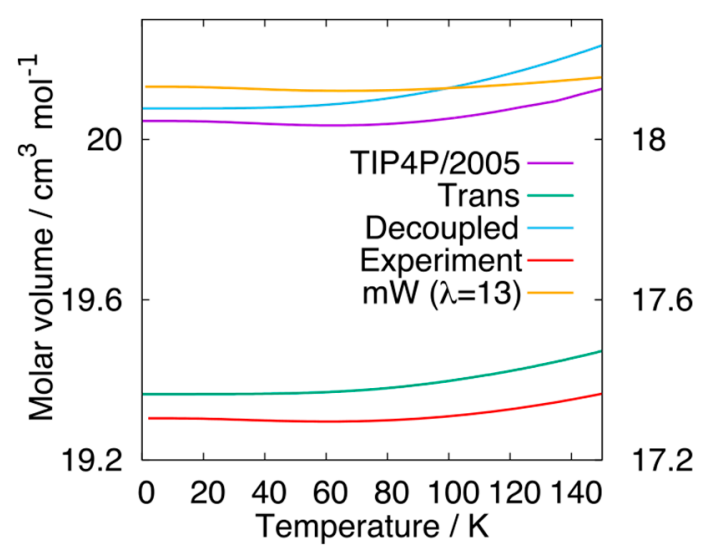

Figure 6. Molar volume of ice Ic plotted against the temperature for the TIP4P/2005 model (purple), that calculated without the rotational-translational coupling (cyan), and that calculated only with the translational modes (green). The red curve is the volume of ice Ih from the experimental data. The volume of the $\mathrm{mW}$ model with $\lambda=13$ is also shown (yellow, right axis).

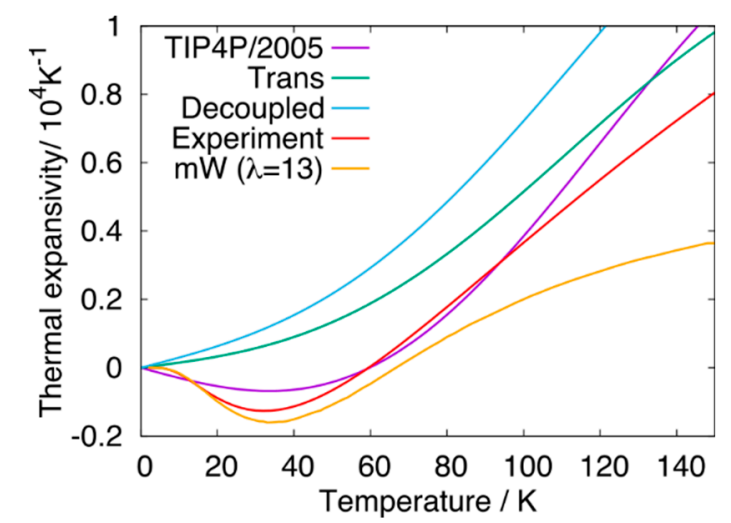

Figure 7. Thermal expansivity of ice Ic plotted against the temperature for the TIP4P/2005 model (purple), that calculated without the rotational-translational coupling (cyan), and that calculated only with the translational modes (green). The red curve is the thermal expansivity from the experimental data [3]. The thermal expansivity of the $\mathrm{mW}$ model with $\lambda=13$ is also shown (yellow).

The thermal expansivity, $\alpha$, can be expressed as:

$$
\alpha=\frac{\gamma C_{v} \kappa_{T}}{3 V}
$$


where $\gamma$ is the Grüneisen parameter, $C_{v}$ is the heat capacity, and $\kappa_{T}$ is the isothermal compressibility. The Grüneisen parameter is expressed as:

$$
\gamma=\sum_{i} \gamma_{i} C_{i} / \sum_{i} C_{i}
$$

where $\gamma_{i}$ and $C_{i}$ are the Grüneisen parameter and heat capacity for the $i$-th mode, respectively. The mode Grüneisen parameter of the $i$-th mode is defined as:

$$
\gamma_{i}=-\left(\frac{\partial \ln v_{i}}{\partial \ln V}\right)
$$

The heat capacity of a mode is given by:

$$
C_{i}=k_{B}\left(\frac{h v_{i}}{k_{B} T}\right)^{2}\left[\exp \left(\frac{h v_{i}}{k_{B} T}\right)-1\right]^{-2} .
$$

Figure 8 shows the frequency dependence of the heat capacities of the vibrational modes defined as:

$$
c(v)=\int_{v}^{v+\Delta v} \sum_{i} \delta\left(v^{\prime}-v\right) C_{i} d v^{\prime},
$$

for the $\mathrm{mW}$ model with $\lambda=13$ at three different temperatures. The heat capacity is almost zero for modes with frequencies higher than $80 \mathrm{~cm}^{-1}$ at $T=20 \mathrm{~K}$, reflecting the fact that only very low-frequency modes can be thermally excited at such a low temperature.

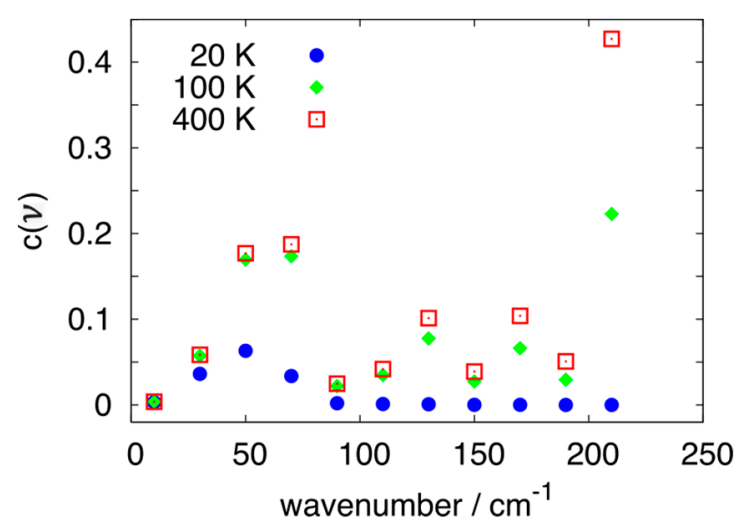

Figure 8. Frequency dependence of the heat capacity of modes at temperatures $20 \mathrm{~K}$ (blue circle), $100 \mathrm{~K}$ (green diamond), and $400 \mathrm{~K}$ (red open square) for the $\mathrm{mW}$ model with $\lambda=13$.

The mode Grüneisen parameter is calculated as follows. First, the mass-weighted Hessian matrix at the equilibrium volume, $\mathbf{K}_{0}$, is diagonalized as:

$$
\boldsymbol{\Lambda}_{0}=\mathbf{U}_{0}^{\dagger} \mathbf{K}_{0} \mathbf{U}_{0},
$$

where $\Lambda_{0}$ is the diagonalized matrix and $\mathbf{U}_{0}$ is the unitary matrix that diagonalizes $\mathbf{K}_{0}$. Next, the volume of the system is scaled isotropically by a factor of 1.0015, and the mass-weighted Hessian for the expanded system, $\mathbf{K}_{e}$, is calculated. Similarly, the original system is scaled by 0.9985 , and the mass-weighted Hessian for the shrunken system, $\mathbf{K}_{s}$, is calculated. The mode frequencies of the expanded and the shrunken systems are calculated with the unitary matrix for the original volume:

$$
\boldsymbol{\Lambda}_{e}=\mathbf{U}_{0}^{\dagger} \mathbf{K}_{e} \mathbf{U}_{0},
$$


and

$$
\Lambda_{s}=\mathbf{U}_{0}^{\dagger} \mathbf{K}_{s} \mathbf{U}_{0} .
$$

The differential value, $\gamma_{i}$, is numerically calculated from $\boldsymbol{\Lambda}_{e}$ and $\boldsymbol{\Lambda}_{s}$. Figure 9 shows the frequency dependence of the product $\gamma_{i} C_{i}$ for the mW model with $\lambda=13$. The $\gamma_{i}$ value is negative for the O-O-O bending modes around $50 \mathrm{~cm}^{-1}$. When the temperature is low enough, only this part contributes to the $\gamma$ defined in Equation (11). At higher temperatures, the positive contributions from the high frequency $\mathrm{O}-\mathrm{O}$ stretching modes become dominant because of the increase in $C_{i}$. Therefore, the negative thermal expansivity is observed only at low temperatures. A similar result was reported for the TIP4P model [6].

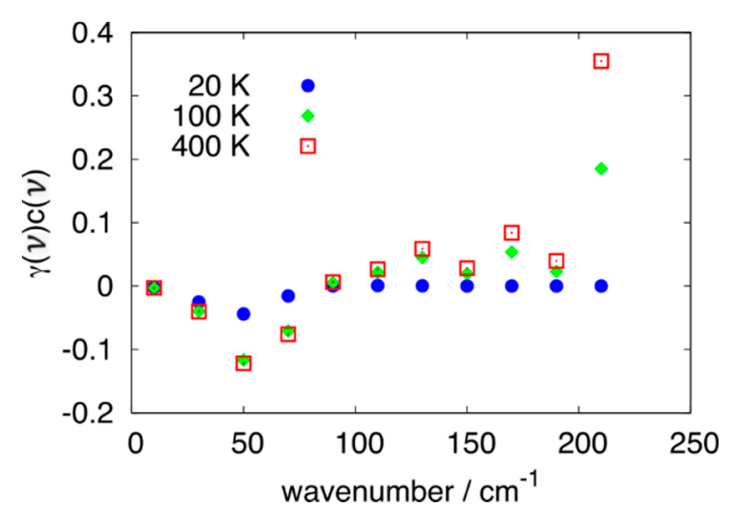

Figure 9. Frequency dependence of $\gamma_{i} C_{i}$ at temperatures $20 \mathrm{~K}$ (blue circle), $100 \mathrm{~K}$ (green diamond), and $400 \mathrm{~K}$ (red open square) for the $\mathrm{mW}$ model with $\lambda=13$.

As shown in Figure 5, the negative thermal expansivity is found for the $\mathrm{mW}$ model when the $\lambda$ parameter is small enough. Figure 10 presents the frequency dependence of $\gamma_{i}$. The mode Grüneisen parameters are positive even in the region around $50 \mathrm{~cm}^{-1}$ for the original $\lambda$ value of 23.15 . This is probably because the modes around $50 \mathrm{~cm}^{-1}$ with the original $\lambda$ value have a lower $\mathrm{O}-\mathrm{O}-\mathrm{O}$ bending character. However, a decrease in the $\lambda$ value can loosen the geometrical restriction of the arrangement on the three neighboring molecules and give rise to rather lower frequency modes, which may have the bending character.

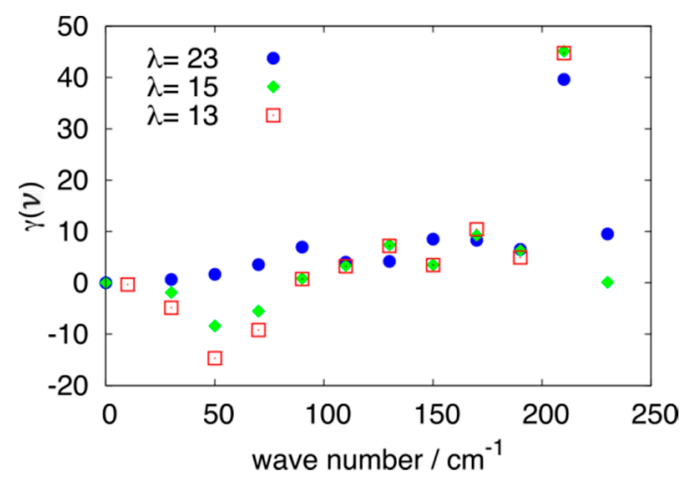

Figure 10. Frequency dependence of the mode Grüneisen parameter for the original $\lambda$ (blue circle), $\lambda=15$ (green diamond), and $\lambda=13$ (red open square).

\section{Conclusions}

We have calculated the chemical potential and volumetric properties of ice polymorphs with the original $\mathrm{mW}$ potential, applying the quasi-harmonic approximation. It is found that only several low-pressure ices (Ih, Ic, II, and III) are stable or metastable with the original mW model. The original $\mathrm{mW}$ model does not reproduce the negative thermal expansivity at low temperatures for ice I. A revision is proposed so as to recover the negative thermal expansivity. It is achieved by reducing one of the 
parameters of the force field model, $\lambda$, which was introduced for three molecules to favor the angle of the ideal tetrahedral arrangement, $109.47^{\circ}$. The origin of the negative thermal expansivity is examined for the revised $\mathrm{mW}$ model in terms of the anomalous mode Grüneisen parameters. In particular, the dependence of the mode Grüneisen parameters on the $\lambda$ value is closely scrutinized. The magnitude of the negative mode Grüneisen parameters generally increases with decreasing $\lambda$ value.

The calculated thermodynamic properties for $\mathrm{mW}$ ice are compared with those of a more realistic model, TIP4P/2005. The origin of the difference in the thermal expansivity is investigated by the chemical potential, whose vibrational free energy part is calculated from the block diagonal matrix in which all off-diagonal elements associated with translational-rotational degrees of freedom are removed. This decoupling of the translational and rotational motions eliminates the anomaly in the thermal expansivity and seems to be related to the observation that the negative thermal expansivity is not recovered with the original $\mathrm{mW}$ potential. The rotational degree of freedom may help to absorb the bare collisional motion between two adjacent oxygens through tight hydrogen bonding. The decrease in the $\lambda$ value in $\mathrm{mW}$, which loosens the geometrical restriction of the arrangement on the three neighboring molecules, seems to have a similar effect.

Author Contributions: M.M.H. performed the simulations and calculations, prepared the data, and wrote the paper; T.Y. co-wrote the paper; M.M. prepared the ice structures and revised the paper; H.T. conducted the project, wrote the programs for normal mode analysis, co-wrote and revised the paper.

Funding: This research was funded by JSPS KAKENHI grant number 17K19106 and MEXT as "Priority Issue on Post-Kcomputer" (Development of new fundamental technologies for high-efficiency energy creation, conversion/storage and use).

Conflicts of Interest: The authors declare no conflict of interest.

\section{References}

1. Dantl, G. Wärmeausdehnung von $\mathrm{H}_{2} \mathrm{O}-$ und $\mathrm{D}_{2} \mathrm{O}$-Einkristallen. Zeitschrift für Physik 1962, 166, 115-118. [CrossRef]

2. Röttger, K.; Endriss, A.; Ihringer, J.; Doyle, S.; Kuhs, W.F. Lattice constants and thermal expansion of $\mathrm{H}_{2} \mathrm{O}$ and $\mathrm{D}_{2} \mathrm{O}$ ice $\mathrm{I} h$ between 10 and $265 \mathrm{~K}$. Addendum. Acta Crystallogr. B 2012, 68, 91. [CrossRef]

3. Fortes, A.D. Accurate and precise lattice parameters of $\mathrm{H}_{2} \mathrm{O}$ and $\mathrm{D}_{2} \mathrm{O}$ ice Ih between 1.6 and $270 \mathrm{~K}$ from high-resolution time-of-flight neutron powder diffraction data. Acta Cryst. 2018, B74, 196-216.

4. Buckingham, D.T.W.; Neumeier, J.J.; Masunaga, S.H.; Yu, Y.K. Thermal Expansion of Single-Crystal $\mathrm{H}_{2} \mathrm{O}$ and $\mathrm{D}_{2} \mathrm{O}$ Ice Ih. Phys. Rev. Lett. 2018, 121, 185505. [CrossRef]

5. Fortes, A.D. Structural manifestation of partial proton ordering and defect mobility in ice Ih. Phys. Chem. Chem. Phys. 2019, 21, 8264-8474. [CrossRef]

6. Tanaka, H. Thermodynamic stability and negative thermal expansion of hexagonal and cubic ices. J. Chem. Phys. 1998, 108, 4887-4893. [CrossRef]

7. Grüneisen, E. Theorie des festen Zustandes einatomiger Elemente. Annalen der Physik 1912, 344, 257-306. [CrossRef]

8. Nakamura, T.; Matsumoto, M.; Yagasaki, T.; Tanaka, H. Thermodynamic Stability of Ice II and Its Hydrogen-Disordered Counterpart: Role of Zero-Point Energy. J. Phys. Chem. B 2016, 120, 1843-1848. [CrossRef]

9. Petrenko, V.F.; Whitworth, R.W. Physics of Ice; Oxford University Press: Oxford, UK, 1999.

10. Grimme, S. Semiempirical GGA-type density functional constructed with a long-range dispersion correction. J. Comput. Chem. 2006, 27, 1787-1799. [CrossRef]

11. Pamuk, B.; Soler, J.M.; Ramìrez, R.; Herrero, C.P.; Stephens, P.W.; Allen, P.B.; Fernàndez-Serra, M.-V. Anomalous Nuclear Quantum Effects in Ice. Phys. Rev. Lett. 2012, 108, 193003. [CrossRef] [PubMed]

12. Salim, M.A.; Willow, S.Y.; Hirata, S. Ice Ih anomalies: Thermal contraction, anomalous volume isotope effect, and pressure-induced amorphization. J. Chem. Phys. 2016, 144, 204503. [CrossRef]

13. Gupta, M.K.; Mittal, R.; Singh, B.; Mishra, S.K.; Adroja, D.T.; Fortes, A.D.; Chaplot, S.L. Phonons and anomalous thermal expansion behavior of $\mathrm{H}_{2} \mathrm{O}$ and $\mathrm{D}_{2} \mathrm{O}$ ice Ih. Phys. Rev. B 2018, 98, 104301. [CrossRef] 
14. McKinley, J.L.; Beran, G.J.O. Identifying pragmatic quasi-harmonic electronic structure approaches for modeling molecular crystal thermal expansion. Faraday Discuss. 2018, 211, 181-207. [CrossRef]

15. Abascal, J.L.F.; Vega, C. A general purpose model for the condensed phases of water: TIP4P/2005. J. Chem. Phys. 2005, 123, 234505. [CrossRef]

16. Sanz, E.; Vega, C.; Abascal, J.L.F.; MacDowell, L.G. Phase Diagram of Water from Computer Simulation. Phys. Rev. Lett. 2004, 92, 255701. [CrossRef] [PubMed]

17. Aragones, J.L.; Vega, C. Plastic crystal phases of simple water models. J. Chem. Phys. 2009, 130, 244504. [CrossRef] [PubMed]

18. Hirata, M.; Yagasaki, T.; Matsumoto, M.; Tanaka, H. Phase Diagram of TIP4P/2005 Water at High Pressure. Langmuir 2017, 33, 11561-11569. [CrossRef] [PubMed]

19. Yagasaki, T.; Matsumoto, M.; Tanaka, H. Phase Diagrams of TIP4P/2005, SPC/E, and TIP5P Water at High Pressure. J. Phys. Chem. B 2018, 122, 7718-7725. [CrossRef] [PubMed]

20. Jorgensen, W.L.; Chandrasekhar, J.; Madura, J.D.; Impey, R.W.; Klein, M.L. Comparison of simple potential functions for simulating liquid water. J. Chem. Phys. 1983, 79, 926-935. [CrossRef]

21. Ramírez, R.; Neuerburg, N.; Fernández-Serra, M.-V.; Herrero, C.P. Quasi-harmonic approximation of thermodynamic properties of ice Ih, II, and III. J. Chem. Phys. 2012, 137, 044502-044513. [CrossRef]

22. Molinero, V.; Moore, E.B. Water modeled as an intermediate element between carbon and silicon. J. Phys. Chem. B 2009, 113, 4008-4016. [CrossRef]

23. Jacobson, L.C.; Hujo, W.; Molinero, V. Nucleation pathways of clathrate hydrates: Effect of guest size and solubility. J. Phys. Chem. B 2010, 114, 13796-13807. [CrossRef]

24. Moore, E.B.; Molinero, V. Ice crystallization in water's "no-man's land". J. Chem. Phys. 2010, $132,244504$. [CrossRef]

25. Stillinger, F.H.; Weber, T.A. Computer simulation of local order in condensed phases of silicon. Phys. Rev. B 1985, 31, 5262-5271. [CrossRef]

26. Laradji, M.; Landau, D.P.; Dünweg, B. Structural properties of $\mathrm{Si}_{1-\mathrm{x}} \mathrm{Ge}_{\mathrm{x}}$ alloys: A Monte Carlo simulation with the Stillinger-Weber potential. Phys. Rev. B Condens. Matter 1995, 51, 4894-4902. [CrossRef]

27. Takenaka, K. Negative thermal expansion materials: Technological key for control of thermal expansion. Sci. Technol. Adv. Mater. 2012, 13, 013001. [CrossRef]

28. Fabian, J.; Allen, P.B. Thermal expansion and Grüneisen parameters of amorphous silicon: A realistic model calculation. Phys. Rev. Lett. 1997, 79, 1885. [CrossRef]

29. Moore, E.B.; Molinero, V. Structural transformation in supercooled water controls the crystallization rate of ice. Nature 2011, 479, 506-508. [CrossRef]

30. Matsumoto, M.; Yagasaki, T.; Tanaka, H. GenIce: Hydrogen-Disordered Ice Generator. J. Comput. Chem. 2018, 39, 61-64. [CrossRef]

31. Li, J. Inelastic neutron scattering studies of hydrogen bonding in ices. J. Chem. Phys. 1996, 105, 6733-6755. [CrossRef]

(C) 2019 by the authors. Licensee MDPI, Basel, Switzerland. This article is an open access article distributed under the terms and conditions of the Creative Commons Attribution (CC BY) license (http://creativecommons.org/licenses/by/4.0/). 